\title{
PLANT TRAITS AND BIOMASS ALLOCATION OF GENTIANA HEXAPHYLLA ON DIFFERENT SLOPE ASPECTS AT THE EASTERN MARGIN OF QINGHAI-TIBET PLATEAU
}

\author{
XUE, J. ${ }^{1,2}-$ HE, J. ${ }^{1,2}-$ WANG, L. ${ }^{1,3}-$ GAO, J. ${ }^{1,4}-$ WU, Y..$^{1^{*}}$ \\ ${ }^{1}$ Chengdu Institute of Biology, Chinese Academy of Sciences, Chengdu 610041, China \\ ${ }^{2}$ University of Chinese Academy of Sciences, Beijing 100049, China \\ ${ }^{3}$ ABA Teachers University, Wenchuan 623002, China \\ ${ }^{4}$ East China Normal University, Shanghai 200241, China \\ *Corresponding author \\ e-mail:wuyan@cib.ac.cn; phone: +86-28-8289-0511; fax:+86-28-8289-0511
}

(Received $14^{\text {th }}$ Nov 2017; accepted $7^{\text {th }}$ Mar 2018)

\begin{abstract}
The growth, biomass partitioning and reproduction of alpine Gentiana hexaphylla species in local microclimates have important ecological significance in the study of the adaptation strategies of alpine plants. At the eastern edge of the Qinghai-Tibet Plateau in China we set up eight sample positions surrounded by mountains at an altitude of $4000 \mathrm{~m}$ and subsequently transplanted and measured the indices of growth, biomass allocation and reproduction of Gentiana hexaphylla to investigate the adaptation strategies in different slope aspects. Our results showed that slope aspects significantly affected the survival and reproduction strategies of Gentiana hexaphylla. Plants growing on sunny slopes tend to reproduce sexually, and those growing on shady slope are inclined to reproduce asexually. Along the circumference of the mountain, from slope S to slope SW, the growth of plant height from the early vegetation stage to the full bloom stage showed a wave shape and biomass accumulation showed a "W"like pattern. The adaption strategy of Gentiana hexaphylla has a stronger correlation with soil organic carbon, soil total nitrogen and phosphorus than with light intensity, soil annual temperature and soil water content. Our results will help to understand the physiological adaptation of Gentiana hexaphylla in different slope aspects and explore the rule of plant functions and character responses to various habitats, so as to reveal the life cycle strategy.
\end{abstract}

Keywords: Gentiana hexaphylla, slope aspect, adaption strategy, growth, biomass allocation, reproduction

\section{Introduction}

Alpine ecosystems, owing to their high altitude, high temperature differences around the clock, and highly varying and major abiotic factors, greatly affect the adaptation and evolution of plants. Ontogenetic modifications and modulation respond to the harsh alpine environment with a high degree of specialization through evolutionary adaptation (Körner, 2003). Embedded in different floras of the world, high mountains often bear greater biodiversity than those in their surrounding lowlands (Körner, 2003; Barthlott et al., 1996). Alpine plants can survive in extreme environments with different reproduction strategies to maintain the stabilization of populations (Stöcklin, 1992; Körner, 2003). Due to their extreme environmental conditions and high biodiversity, high mountain ecosystems are an ideal region for studying plant adaptations (Sun et al., 2014). Studying the growth traits, biomass allocation and reproduction of plants in severe environments is conductive to understanding the reasons for the biodiversity of high mountain ecosystems. As a typical fragile and sensitive ecosystem, plant structure 
and functional trait responses to different habitats were helpful for understanding the mechanisms of plant adaptation to extreme environments and predicting vegetation change under the background of climate change (Schneider, 1993; IPCC, 2001).

Topography is a comprehensive indicator of habitat conditions that can create local microclimates (Cantón et al., 2004). As a vital topographic factor, slope aspect affects the daily cycle of solar radiation and has a strong influence on aspects of the microclimate, especially air and soil temperature, soil moisture and soil nutrition (Rosenberg et al., 1983; Fekedulegn et al., 2003; Auslander et al., 2003). Soil temperature and moisture significantly influence soil nutrient mineralization in different slope aspects (Sternberg and Shoshany, 2001; Ai et al., 2017). Previous studies found that the spatial variation in slope aspect was a determinant element for community structure, species diversity, ecosystem processes and plant functional traits (De Bello et al., 2006; Bennie et al., 2008; Carletti et al., 2009). Along the circumference of the mountain, the spatial variation in slope aspect displays a natural habitat gradient. Due to the complexity of the ecological environment and the specialization of alpine plants, it is important to conduct research on different slope aspects in alpine ecosystems.

Plants inhabiting harsh environments have evolved highly specialized phenological, morphological and physiological mechanisms and structures (Caldwell, 1968, 1979; Nagy and Grabherr, 2009). Plant height (PH) and leaf number are indicators of plant interactions with their environment (Grassein et al., 2010), and biomass partitioning of plants drives carbon assimilation and nutrient absorption (Poorter et al., 2012). Specific leaf area (SLA) can show large variability in response to irradiance, temperature, and water availability (Pigliucci and Kolodynska, 2002; Poorter et al., 2009). Numerous studies have explored plant ecological adaptions and reproductive mechanisms (Ohba, 1988; He et al., 2005; Yang and Sun, 2006; Peng et al., 2012). Flower structure traits indicate the ability of sexual reproduction (Mcintosh, 2002), while the branch numbers of plants indicate the ability for asexual reproduction (He, 2017). Allometric theory has been used increasingly in the relationship between plant plasticity response and plant functional traits (Weiner, 2004; Bonser and Aarssen, 2009). The change in habitat conditions caused by slope aspect deeply affects plant growth rhythm; to understand how plant functional traits respond to the topographic environment, the important ecological factors must be identified by researching the allometric growth relationships among plant traits for plants growing in different slope aspects. There are many studies on the effects of elevation on plant adaption, and several slope orientation studies have discussed the different effects of sunny vs shady slope position on alpine plant growth, but there is very little research concerning the effect of slope aspect along a continuous mountain level-curve ring. Gentiana hexaphylla, a perennial herbaceous species, grows well in the harsh alpine environment and distributed in all slope aspects around the mountains, but its growth differs. These plants reproduce both sexually and vegetatively from rhizomes (Bynum, 2001). At the same time, the flower of Gentiana hexaphylla was larger than that of other herbs at the sample sites, which was easy to observe. Different slope aspects are due mainly to the distribution of hydrothermal condition. There is a lack of research on the growth traits, biomass partitioning and reproduction of this taxon, and we have studied the effect of elevation (He et al., 2017) and now discuss the influence of slope aspects to make the research more systematic. We set 8 sites to investigate the different survival strategies on different slope aspects on a relatively isolated mountain. We proposed several hypotheses: (1) slope aspects significantly affected plant growth, biomass allocation and reproduction strategies; (2) along the 
circumference of the mountain, the increasing of plant height and the increment of biomass from vegetation stage to full bloom stage were significantly affected by slope aspects; and (3) Gentiana hexaphylla grew on different slope aspects using different reproduction strategies to ensure the survival of individuals and the continuation of populations. Proposing these hypotheses will further our understanding of the physiological adaptive ability of Gentiana hexaphylla in different slope aspects and will clarify the patterns of responses to different habitats among plant traits.

\section{Materials and methods}

\section{Site characteristics}

The study area was located in Snow Ridge $\left(32^{\circ} 44^{\prime} 42^{\prime \prime} \sim 32^{\circ} 44^{\prime} 46^{\prime \prime} \quad \mathrm{N}\right.$, $103^{\circ} 43^{\prime} 44^{\prime \prime} \sim 103^{\circ} 43^{\prime} 49^{\prime \prime}$ E) in Songpan County, Sichuan Province, at the eastern edge of the Qinghai-Tibet Plateau in China (Figs. 1 and 2). The average altitude of the study area is 4,005 4,074 m. Snow Ridge belongs to the Minshan mountain system. The climate of the study area is characterized by a long winter and a short summer. This region has the typical humid and semi-humid continental monsoon climate of the plateau cold temperate zone. It has a warm rainy season, and the air temperature varies significantly from day to night. The zone has a mean annual temperature of $2.8^{\circ} \mathrm{C}$ and a mean month temperature from $-7.6{ }^{\circ} \mathrm{C}$ in January to $9.7{ }^{\circ} \mathrm{C}$ in July, and there is no absolutely frost-free period. The mean annual solar radiation hours are $1827.5 \mathrm{~h}$, and the average annual precipitation is $634.8 \mathrm{~mm}$. The yearly accumulated temperature above $10{ }^{\circ} \mathrm{C}$ is $428.6{ }^{\circ} \mathrm{C}$ (Chen, 2011). The soil belongs to the mountain brown meadow group. Vegetation is the alpine meadow type (Chen, 2008).

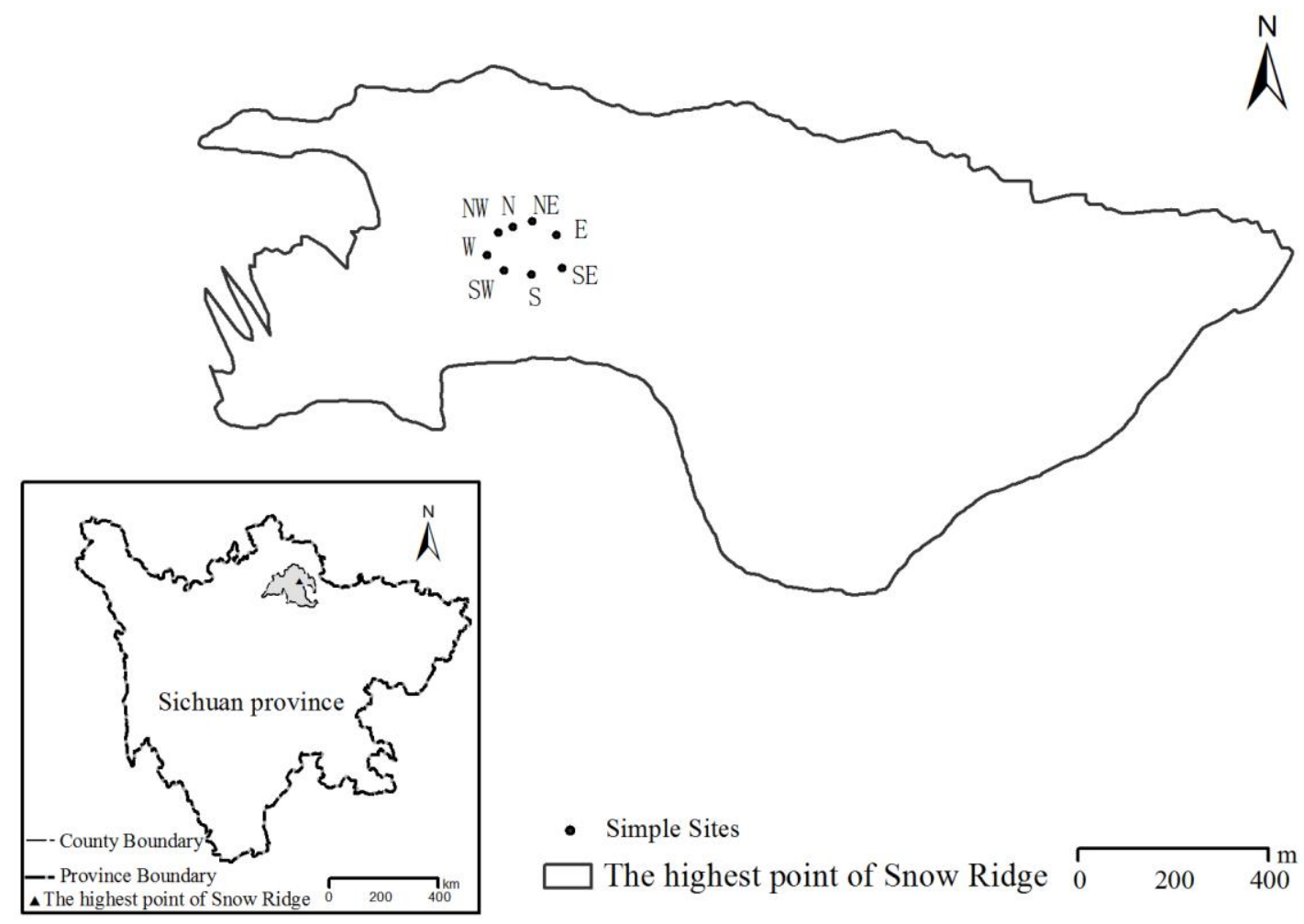

Figure 1. Map of the location of sample sites 

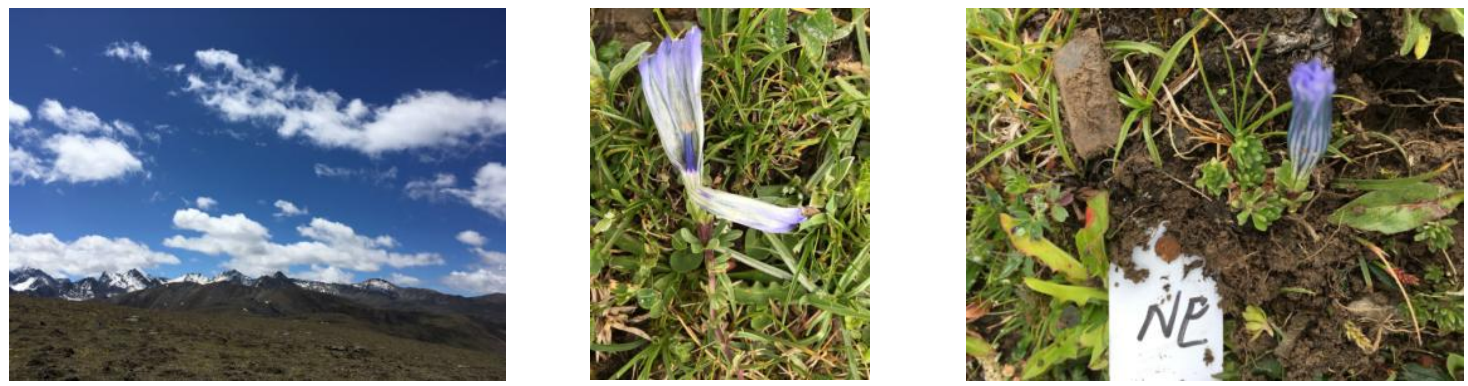

Figure 2. Habitat photos

\section{Materials}

Gentiana hexaphylla, distributed in alpine meadows, grassland and roadside slopes (2700-4400 m), is a perennial herbaceous species that develops two or three flowering shoots and a single terminal flower on each flowering shoot. Roots are mostly slightly fleshy and fibrous. Stems are clustered, diffuse, obliquely ascending, and papillate. Basal rosette leaves are absent or poorly developed. The calyx tube is narrowly obconic and the corolla is blue with a pale yellow-white base and dark blue streaks. Stamens are inserted at the basal part of the corolla tube, and anthers are narrowly ellipsoid. Stigma lobes are oblong, and capsules are ovoid-ellipsoid. Seeds are light brown and ellipsoid to ovoid. The flowering and fruiting period lasts from July to September.

\section{Experimental design}

One relatively isolated mountain was selected as the area of study. We set up 8 sample positions as sites on the southern (S), southeast (SE), east (E), northeast (NE), northern $(\mathrm{N})$, northwest $(\mathrm{NW})$, west $(\mathrm{W})$, and southwest (SW) slopes along the circumference of mountain. The target plants were selected and fixed beginning in May 2016 after the snow melt. We dug up the whole plant (complete with roots) along with the original soil $(10 \mathrm{~cm} \times 10 \mathrm{~cm})$ to ensure that the target plant was located in the centre position. We chose 800 plants with similar heights that were growing well, and then moved and fixed them to the 8 sites (100 plants per transplant site). Plants were then placed in a ditch that was dug before the experiment. The surrounding soil was used to ensure that the sample plants were flush with the ground. The plant samples were fixed at $50 \mathrm{~cm}$ intervals for the follow-up experiment. The plants (Gentiana hexaphylla) were transplanted to each of the 8 sites according to the above method.

\section{Sample collection and treatment in the laboratory}

At the full bloom stage in 2017, 30 individual flowering plants were randomly selected from each of the 8 sites along the slope. Fresh leaf samples were scanned by a flatbed scanner (CanoScan5600F, Canon, Tokyo, Japan), and then Photoshop CS4 and Matlab 7.9 were used to calculate the leaf area (Vile et al., 2005). Each sample was placed in an oven at $65{ }^{\circ} \mathrm{C}$ and dried to constant weight, and dried samples were weighed with an electrical balance of $1 / 10000 \mathrm{~g}$ resolution. At each sample position, three transplantable original soil samples were taken and sieved $(<2 \mathrm{~mm})$; roots and visible organic debris were removed by hand. Light intensity was measured by a digital illuminance meter (TES-1332A) at 10: 00 every day during the flower stage. The surface soil temperature $(10 \mathrm{~cm}$ ) was measured by an RTE (iButton-TMEX). Soil water 
content was measured by the oven drying method. Approximately $100 \mathrm{~g}$ of each soil sample was air-dried for analysis of soil properties (e.g., C, N, and P). Soil organic carbon was determined by potassium dichromate oxidation (Allen, 1989). The total nitrogen was determined by the Kjeldahl method (Allen, 1989), and the vanadium molybdenum yellow colorimetric method was used for the determination of total phosphorus (Bao, 2000).

\section{Statistical analysis}

One-way ANOVA was used to compare the differences in soil water content, soil organic carbon, total nitrogen, total phosphorus, plant height, branch number, leaf number, specific leaf area and biomass of different plant structures among sample positions. Principal component analysis (PCA) was conducted to demonstrate the effects of abiotic factors on plant traits and biomass allocation. Figures were drawn using Origin 9.0. All statistical analyses were conducted using SPSS 19.0 (SPSS Inc., Chicago, IL, USA). Differences were considered significant when $P<0.05$.

The relationships between aboveground biomass and belowground biomass, reproductive biomass and vegetative biomass, and photosynthetic biomass and non- photosynthetic biomass were analysed using the allometric model $\mathrm{y}=\mathrm{ax}{ }^{\mathrm{b}}$. The formula is expressed $\operatorname{logarithmically}$ as $\log \mathrm{y}=\log \mathrm{a}+\mathrm{b} \log \mathrm{x}(\mathrm{x}$ and $\mathrm{y}$ represent biomass, $\mathrm{a}$ is $\mathrm{a}$ standardized constant, and $b$ is the allometric growth index) (Niklas, 2005). Standardized major axis (SMA) slope-fitting techniques were used to calculate the slope (b) and intercept (log a) of the allometric growth equation (Wright et al., 2001; Warton et al., 2006). The above analysis was conducted in SMART Version 2.

\section{Results}

\section{Environmental factors of different slope aspects}

The daily average temperature of increasing and decreasing temperature was higher on slopes S, SE, and SW than on slopes NW, NE, and N during the four stages of plant growth. Meanwhile, slopes E and W also showed a higher temperature than that on slopes NW, NE, and N (Fig. 3). There was no significant difference in light intensity among sites $(\mathrm{F}=0.776, p=0.616)$. The differences in mean annual temperature were significant $(\mathrm{F}=34.886, p<0.001)$. The mean annual temperature of slope $\mathrm{E}$ was significantly greater than those of the sunny slopes and slope $\mathrm{W}$, while the temperatures of the sunny slopes and slope $\mathrm{W}$ were significantly greater than the temperatures of the shady slopes. There was a significant difference in soil water content among sites $(\mathrm{F}=4.445, p=0.006)$. Slope $\mathrm{N}$ had the highest soil water content $(43.54 \%)$, but slope NW had the lowest (34.38\%), and the difference was significant. At the same time, the soil water content of slope $\mathrm{N}$ was significantly higher than that of slopes $\mathrm{S}$ and $\mathrm{E}$. There were significant differences in soil organic carbon (SOC), soil total nitrogen (STN), and soil total phosphorus (STP) among sites $(\mathrm{F}=4.919, p=0.004 ; \mathrm{F}=4.826, p=0.004$; $\mathrm{F}=8.859, p<0.001$, respectively). Overall, the soil organic carbon, total nitrogen and phosphorus of slope $\mathrm{W}$ were the highest, and those of slope $\mathrm{S}$ were lowest (Table 1).

\section{Morphological traits at the full bloom stage under different slope aspects}

Plant height $(\mathrm{F}=4.909, \mathrm{P}<0.001)$, branch number $(\mathrm{F}=2.231, \mathrm{P}=0.033)$, specific leaf area $(\mathrm{F}=9.958, \mathrm{P}<0.001)$ and leaf number $(\mathrm{F}=2.601, \mathrm{P}=0.013)$ were 
significantly different among sites. The plant heights on slopes $\mathrm{S}$ and $\mathrm{E}$ were remarkably higher than those on the other five slope aspects, except for slope NW. Branch number on slope $\mathrm{N}$ was the largest and significantly larger than those on slopes $\mathrm{NW}, \mathrm{NE}, \mathrm{E}$ and $\mathrm{W}$. The specific leaf area on slope SW was significantly higher than those on the other slope aspects. The leaf number on slopes SW and NE were significantly greater than those on slopes NW and W (Fig. 4, Table 2).
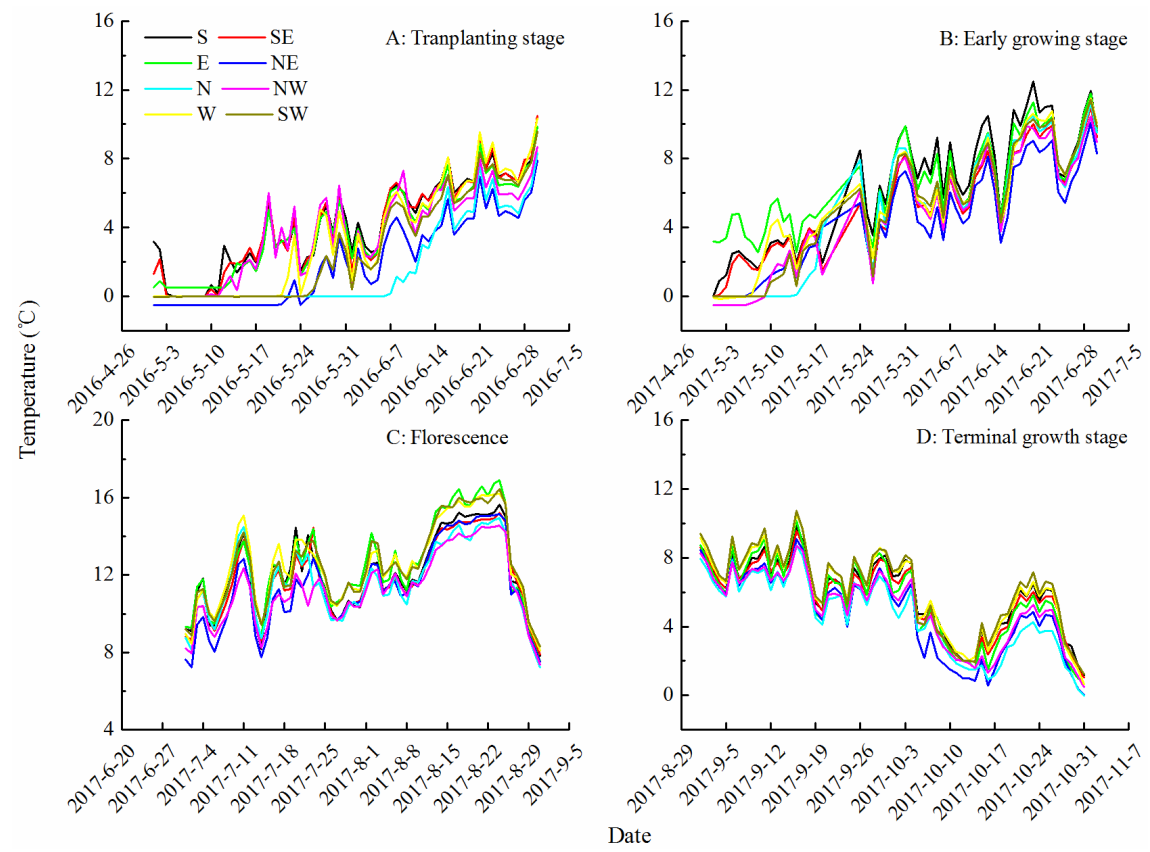

Figure 3. Daily mean soil temperature at sample position. A, B, C, and D represent the different stages of plant growth. $S, S E, E, N E, N, N W, W$, and $S W$ represent the eight different slope aspects
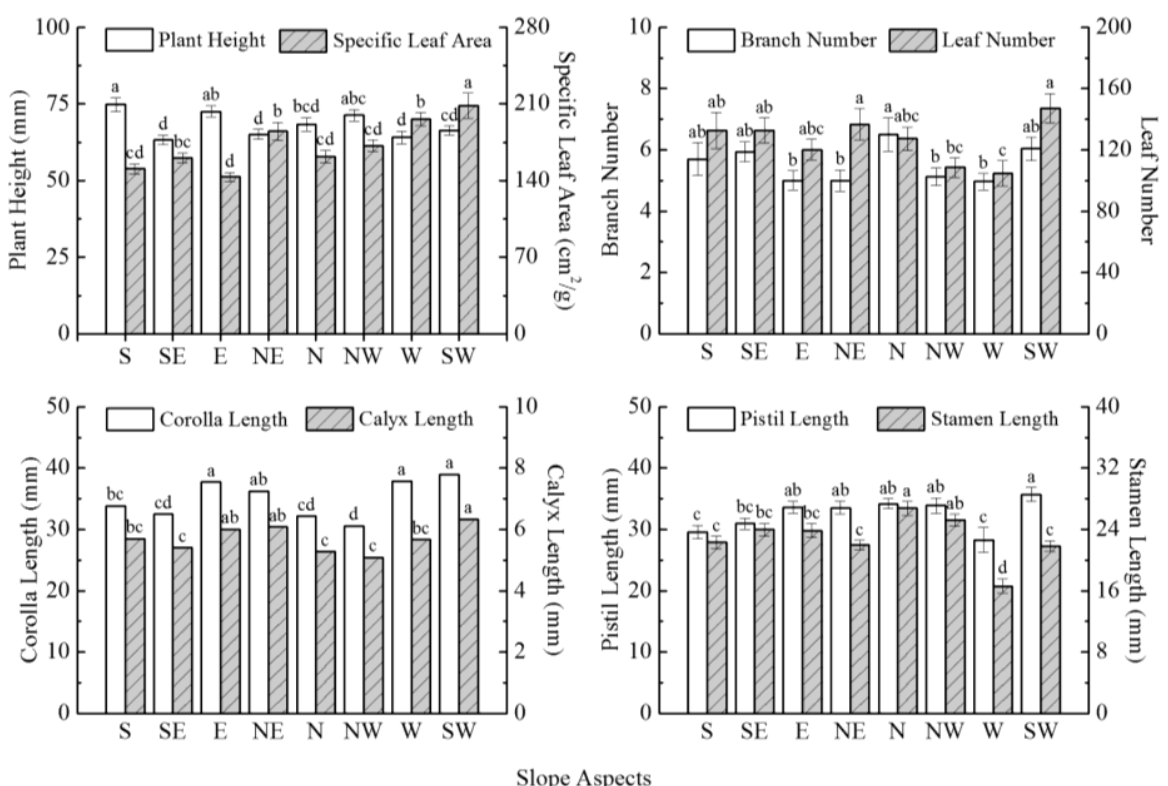

Figure 4. Plant height, specific leaf area, branch number, leaf number and flower traits on different slope aspects. The data were represent the mean $\pm S E(n=30)$ 
Table 1. Comparison of environmental traits among different sampling sites

\begin{tabular}{|c|c|c|c|c|c|c|c|c|}
\hline $\begin{array}{l}\text { Sample } \\
\text { position }\end{array}$ & Latitude and longitude & $\begin{array}{l}\text { Altitude } \\
\text { (m) }\end{array}$ & $\begin{array}{c}\text { Light intensity } \\
(20000 \text { lux })\end{array}$ & $\begin{array}{c}\text { Mean annual } \\
\text { temperature }\left({ }^{\circ} \mathrm{C}\right)\end{array}$ & $\begin{array}{c}\text { Soil water } \\
\text { content }(\%)\end{array}$ & $\begin{array}{c}\text { Soil organic } \\
\text { carbon }(\mathbf{g} / \mathbf{k g})\end{array}$ & $\begin{array}{c}\text { Soil total nitrogen } \\
(\mathrm{g} / \mathrm{kg})\end{array}$ & $\begin{array}{c}\text { Soil total } \\
\text { phosphorus }(\mathrm{g} / \mathrm{kg})\end{array}$ \\
\hline $\mathrm{S}$ & $\mathrm{N} 32^{\circ} 44^{\prime} 42^{\prime \prime} \mathrm{E} 103^{\circ} 43^{\prime} 47^{\prime \prime}$ & 4074 & $975.00 \pm 36.66$ & $3.24 \pm 0.04^{b}$ & $38.81 \pm 0.23^{\mathrm{b}}$ & $29.57 \pm 0.46^{\mathrm{d}}$ & $2.69 \pm 0.06^{\mathrm{d}}$ & $0.61 \pm 0.01^{\mathrm{d}}$ \\
\hline SE & N $32^{\circ} 44^{\prime} 43^{\prime \prime}$ E $103^{\circ} 43^{\prime} 49^{\prime \prime}$ & 4070 & $954.33 \pm 46.00$ & $3.06 \pm 0.13^{\mathrm{b}}$ & $39.16 \pm 3.02^{\mathrm{ab}}$ & $31.95 \pm 2.94^{\mathrm{cd}}$ & $2.80 \pm 0.22^{\mathrm{cd}}$ & $0.68 \pm 0.04^{\mathrm{cd}}$ \\
\hline $\mathrm{NE}$ & N $32^{\circ} 44^{\prime} 46^{\prime \prime}$ E $103^{\circ} 43^{\prime} 47^{\prime \prime}$ & 4050 & $894.33 \pm 95.63$ & $1.81 \pm 0.00^{\mathrm{d}}$ & $40.48 \pm 0.52^{\mathrm{ab}}$ & $33.66 \pm 0.45^{\mathrm{a}}$ & $2.93 \pm 0.07^{\mathrm{bcd}}$ & $0.66 \pm 0.01^{\mathrm{cd}}$ \\
\hline $\mathrm{N}$ & $\mathrm{N} 32^{\circ} 44^{\prime} 46^{\prime \prime} \mathrm{E} 103^{\circ} 43^{\prime} 45^{\prime \prime}$ & 4005 & $884.00 \pm 86.12$ & $2.20 \pm 0.24^{\mathrm{c}}$ & $43.54 \pm 0.68^{\mathrm{a}}$ & $37.03 \pm 0.90^{\mathrm{ab}}$ & $3.30 \pm 0.07^{\mathrm{ab}}$ & $0.79 \pm 0.02^{\mathrm{ab}}$ \\
\hline NW & N $32^{\circ} 44^{\prime} 45^{\prime \prime}$ E $103^{\circ} 43^{\prime} 44^{\prime \prime}$ & 4010 & $848.00 \pm 123.44$ & $2.32 \pm 0.01^{\mathrm{c}}$ & $34.38 \pm 0.83^{\mathrm{c}}$ & $34.33 \pm 1.08^{\mathrm{bc}}$ & $2.79 \pm 0.12^{\mathrm{cd}}$ & $0.68 \pm 0.04^{\mathrm{cd}}$ \\
\hline SW & N $32^{\circ} 44^{\prime} 43^{\prime \prime}$ E $103^{\circ} 43^{\prime} 45^{\prime \prime}$ & 4061 & $951.67 \pm 62.01$ & $3.09 \pm 0.28^{\mathrm{b}}$ & $40.56 \pm 1.14^{\mathrm{ab}}$ & $33.37 \pm 1.57^{\mathrm{bcd}}$ & $3.16 \pm 0.14^{\mathrm{abc}}$ & $0.82 \pm 0.03^{\mathrm{a}}$ \\
\hline $\mathrm{F}$ & & & 0.776 & 34.886 & 3.853 & 4.919 & 4.826 & 8.859 \\
\hline$p$ & & & 0.616 & $<0.001$ & 0.012 & 0.004 & 0.004 & $<0.001$ \\
\hline
\end{tabular}

The data represent the mean $\pm \mathrm{SE}(\mathrm{n}=3)$. Data with the same letters within the same column indicate no significant difference at the $p<0.05$ level. The same below.

Table 2. Effects of slope aspect on plant height, branch number, specific leaf area, leaf number and flower traits

\begin{tabular}{|c|c|c|c|c|c|c|c|c|}
\hline Slope aspect & $\begin{array}{c}\text { Plant height } \\
\text { (mm) }\end{array}$ & Branch number & $\begin{array}{l}\text { Specific leaf area } \\
\left(\mathrm{cm}^{2} \mathrm{~g}^{-1}\right)\end{array}$ & Leaf number & $\begin{array}{c}\text { Corolla length } \\
(\mathbf{m m})\end{array}$ & $\begin{array}{l}\text { Calyx length } \\
(\mathbf{m m})\end{array}$ & $\begin{array}{c}\text { Pistil length } \\
\text { (mm) }\end{array}$ & $\begin{array}{c}\text { Stamen length } \\
(\mathrm{mm})\end{array}$ \\
\hline $\mathrm{S}$ & $74.77 \pm 2.30^{c}$ & $5.70 \pm 0.54^{\mathrm{ab}}$ & $150.72 \pm 4.66^{\mathrm{a}}$ & $132.50 \pm 11.77^{\mathrm{a}}$ & $33.87 \pm 0.10^{\mathrm{bc}}$ & $5.70 \pm 0.02^{\mathrm{bc}}$ & $29.59 \pm 1.07^{\mathrm{c}}$ & $22.31 \pm 0.81^{\mathrm{c}}$ \\
\hline SE & $63.23 \pm 1.58^{\mathrm{ab}}$ & $5.93 \pm 0.33^{\mathrm{ab}}$ & $160.83 \pm 4.23^{b c}$ & $132.63 \pm 8.29^{\mathrm{b}}$ & $32.46 \pm 0.10^{\mathrm{cd}}$ & $5.40 \pm 0.02^{\mathrm{c}}$ & $30.93 \pm 0.96^{\mathrm{bc}}$ & $23.98 \pm 0.84^{b c}$ \\
\hline $\mathrm{E}$ & $72.50 \pm 1.90^{\mathrm{abc}}$ & $5.00 \pm 0.31^{\mathrm{b}}$ & $143.35 \pm 3.96^{b c}$ & $120.17 \pm 6.59^{\mathrm{c}}$ & $37.76 \pm 0.09^{\mathrm{a}}$ & $6.01 \pm 0.02^{\mathrm{d}}$ & $33.61 \pm 0.98^{\mathrm{ab}}$ & $23.83 \pm 0.91^{\mathrm{bc}}$ \\
\hline $\mathrm{NE}$ & $65.03 \pm 1.65^{\mathrm{bc}}$ & $5.00 \pm 0.35^{\mathrm{b}}$ & $184.92 \pm 8.38^{\mathrm{c}}$ & $136.67 \pm 10.23^{\mathrm{c}}$ & $36.26 \pm 0.09^{\mathrm{ab}}$ & $6.09 \pm 0.01^{\mathrm{ab}}$ & $33.53 \pm 1.06^{\mathrm{ab}}$ & $21.99 \pm 0.58^{c}$ \\
\hline $\mathrm{N}$ & $68.30 \pm 2.18^{\mathrm{abc}}$ & $6.50 \pm 0.55^{\mathrm{a}}$ & $162.01 \pm 5.38^{\mathrm{bc}}$ & $127.20 \pm 7.78^{\mathrm{c}}$ & $32.16 \pm 0.06^{\mathrm{cd}}$ & $5.28 \pm 0.02^{\mathrm{c}}$ & $34.18 \pm 0.84^{\mathrm{ab}}$ & $26.75 \pm 0.91^{\mathrm{a}}$ \\
\hline NW & $71.27 \pm 1.84^{\mathrm{a}}$ & $5.13 \pm 0.29^{b}$ & $171.93 \pm 5.13^{\mathrm{abc}}$ & $108.47 \pm 6.21^{\mathrm{c}}$ & $30.55 \pm 0.10^{\mathrm{d}}$ & $5.09 \pm 0.02^{\mathrm{c}}$ & $33.87 \pm 1.30^{\mathrm{ab}}$ & $25.19 \pm 0.79^{\mathrm{ab}}$ \\
\hline W & $64.04 \pm 2.12^{\mathrm{c}}$ & $4.96 \pm 0.27^{\mathrm{b}}$ & $196.09 \pm 5.94^{\mathrm{ab}}$ & $104.67 \pm 8.22^{\mathrm{c}}$ & $37.89 \pm 0.09^{\mathrm{a}}$ & $5.67 \pm 0.02^{\mathrm{bc}}$ & $28.28 \pm 2.01^{\mathrm{c}}$ & $16.61 \pm 0.94^{\mathrm{d}}$ \\
\hline SW & $66.27 \pm 1.57^{\mathrm{bc}}$ & $6.03 \pm 0.38^{\mathrm{ab}}$ & $208.07 \pm 11.64^{\mathrm{a}}$ & $147.17 \pm 9.57^{\mathrm{b}}$ & $38.96 \pm 0.10^{\mathrm{a}}$ & $6.33 \pm 0.02^{\mathrm{a}}$ & $35.71 \pm 1.13^{\mathrm{a}}$ & $21.83 \pm 0.71^{\mathrm{c}}$ \\
\hline $\mathrm{F}$ & 4.909 & 2.231 & 9.958 & 2.601 & 11.685 & 4.735 & 4.367 & 12.803 \\
\hline$p$ & $<0.001$ & 0.033 & $<0.001$ & 0.013 & $<0.001$ & $<0.001$ & $<0.001$ & $<0.001$ \\
\hline
\end{tabular}

The data represent the mean $\pm \mathrm{SE}(\mathrm{n}=30)$. Data with the same letters within the same column indicate no significant difference at the $p<0.05$ level. 
Corolla length $(\mathrm{F}=11.685, \mathrm{P}<0.001)$, calyx length $(\mathrm{F}=4.735, \mathrm{P}<0.001)$, pistil length $(\mathrm{F}=4.367, \mathrm{P}<0.001)$ and stamen length $(\mathrm{F}=12.803, \mathrm{P}<0.001)$ were significantly different among the eight slope aspects. The corolla length of plants grown on slope SW was the largest and was significantly greater than that of plants grown on slopes NE, S, SE, N and NW, the calyx length on slope SW was the largest and was significantly greater than that of that on slopes S, W, SE, N and NW; the pistil length on slope SW was significantly greater than that on slopes SE, S and W; and the stamen length on slopes $\mathrm{N}$ and $\mathrm{NW}$ were significantly greater than those on the other slopes (Table 2).

\section{Biomass allocation of organs and allometric relationships on different slope aspects}

Root biomass allocation was significantly different among the eight slope aspects ( $\mathrm{F}$ $=10.511, p<0.001)$; root biomass allocation on slopes $\mathrm{W}$ and $\mathrm{N}$ were significantly larger than that on other slope aspects. Stem biomass allocation was significantly different among the eight slope aspects $(\mathrm{F}=9.228, p<0.001)$; the stem biomass allocation on slopes $\mathrm{S}, \mathrm{SW}$ and $\mathrm{E}$ were significantly greater than that on slopes NW, SE, $\mathrm{N}$ and $\mathrm{W}$, and that on slope $\mathrm{W}$ was remarkably lower than those on the other slope aspects. Leaf biomass allocation did not remarkably among slope aspects ( $\mathrm{F}=1.295, p$ $=0.254$ ). Flower biomass allocation was not significantly different among the eight slope aspects $(\mathrm{F}=1.390, p=0.211)$. The ratio of belowground biomass/aboveground biomass was significantly different among the eight slope aspects $(\mathrm{F}=16.165, p<$ 0.001); the ratio on slope $\mathrm{W}$ was significantly larger than that on the other slope aspects, and the ratio on slope $\mathrm{N}$ was remarkably greater than that on slope $\mathrm{S}$ (Fig. 5, Table 3).

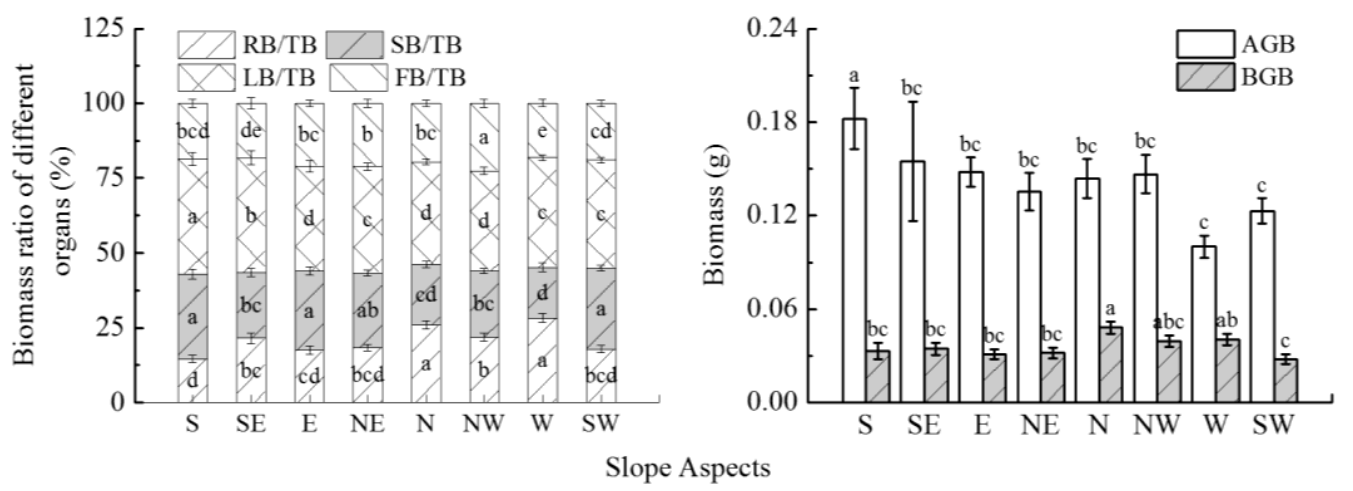

Figure 5. Biomass allocation on different slope aspects. $R B / T B$ represents root biomass allocation, $S B / T B$ represents stem biomass allocation, $L B / T B$ represents leaf biomass

allocation, FB/TB represents flower biomass allocation, $A G B$ represents aboveground biomass, and $B G B$ represents belowground biomass. The data represent the mean $\pm S E(n=30)$

There was a significant difference in the belowground/aboveground biomass relationships among different slope aspects (WALD $=18.672, p=0.009$ ). The relationship between belowground and aboveground growth was allometric. There were no significant differences between reproductive/vegetative biomass relationships among different slope aspects (WALD $=8.564, p=0.267$ ). The allometric relationship among the 8 sites had a common slope of 1.007 , which was not significantly different from 1 (chi square test: $\chi^{2}=0.015, p=0.903$ ). The relationship between reproductive and vegetative growth was isometric. Further examination showed that the intercepts of 
reproductive and vegetative biomass in different slopes were not significantly different, and there was no significant coaxial drift. There was a significant difference between photosynthetic/non-photosynthetic biomass relationships among the different slope aspects (WALD $=17.394, p=0.019$ ). The relationship between photosynthetic and non-photosynthetic growth was allometric (Table 4).

Table 3. Biomass allocation and root/shoot ratio of plants on different slope aspects

\begin{tabular}{c|c|c|c|c|c}
\hline Sample & $\mathbf{R B} / \mathbf{T B}$ & $\mathbf{S B} / \mathbf{T B}$ & $\mathbf{L B} / \mathbf{T B}$ & $\mathbf{F B} / \mathbf{T B}$ & $\mathbf{B G B} / \mathbf{A G B}$ \\
\hline $\mathrm{S}$ & $0.15 \pm 0.01^{\mathrm{d}}$ & $0.28 \pm 0.02^{\mathrm{a}}$ & $0.38 \pm 0.02$ & $0.19 \pm 0.01$ & $0.75 \pm 0.07^{\mathrm{e}}$ \\
$\mathrm{SE}$ & $0.22 \pm 0.02^{\mathrm{bc}}$ & $0.22 \pm 0.01^{\mathrm{bc}}$ & $0.38 \pm 0.02$ & $0.18 \pm 0.02$ & $1.09 \pm 0.11^{\mathrm{bc}}$ \\
$\mathrm{E}$ & $0.17 \pm 0.01^{\mathrm{cd}}$ & $0.27 \pm 0.01^{\mathrm{a}}$ & $0.35 \pm 0.02$ & $0.21 \pm 0.01$ & $0.69 \pm 0.04^{\mathrm{de}}$ \\
$\mathrm{NE}$ & $0.18 \pm 0.01^{\mathrm{bcd}}$ & $0.25 \pm 0.01^{\mathrm{ab}}$ & $0.36 \pm 0.01$ & $0.21 \pm 0.01$ & $0.70 \pm 0.11^{\mathrm{cde}}$ \\
$\mathrm{N}$ & $0.26 \pm 0.01^{\mathrm{a}}$ & $0.20 \pm 0.01^{\mathrm{cd}}$ & $0.34 \pm 0.01$ & $0.20 \pm 0.01$ & $1.38 \pm 0.11^{\mathrm{b}}$ \\
$\mathrm{NW}$ & $0.22 \pm 0.01^{\mathrm{b}}$ & $0.22 \pm 0.01^{\mathrm{bc}}$ & $0.33 \pm 0.01$ & $0.23 \pm 0.01$ & $0.95 \pm 0.13^{\mathrm{bcd}}$ \\
$\mathrm{W}$ & $0.28 \pm 0.01^{\mathrm{a}}$ & $0.17 \pm 0.02^{\mathrm{d}}$ & $0.37 \pm 0.01$ & $0.18 \pm 0.01$ & $1.49 \pm 0.18^{\mathrm{a}}$ \\
$\mathrm{SW}$ & $0.18 \pm 0.01^{\mathrm{bcd}}$ & $0.27 \pm 0.01^{\mathrm{a}}$ & $0.36 \pm 0.01$ & $0.19 \pm 0.01$ & $0.65 \pm 0.04^{\mathrm{de}}$ \\
$\mathrm{F}$ & 10.511 & 9.228 & 1.295 & 1.390 & 16.165 \\
$p$ & $<0.001$ & $<0.001$ & 0.254 & 0.211 & $<0.001$ \\
\hline
\end{tabular}

$\mathrm{RB} / \mathrm{TB}, \mathrm{SB} / \mathrm{TB}, \mathrm{LB} / \mathrm{TB}$, and $\mathrm{FB} / \mathrm{TB}$ represent root, stem, leaf, and flower biomass partitioning, respectively. $\mathrm{BGB} / \mathrm{AGB}$ represents aboveground/belowground biomass. The data represent the mean \pm $\mathrm{SE}(\mathrm{n}=30)$. Data with the same letters within the same column indicate a not significant difference at the $p<0.05$ level.

Table 4. Allometric scaling exponents and the test of isometry between plant functional traits on different slope aspects

\begin{tabular}{l|c|c|c|c|c|c|c}
\hline \multicolumn{2}{c|}{$\begin{array}{c}\text { Sample } \\
\text { position }\end{array}$} & $\mathbf{R}^{2}$ & $\boldsymbol{p}$ & $\begin{array}{c}\text { Slope (95\% confidence } \\
\text { interval) }\end{array}$ & $\begin{array}{c}\text { Intercept (95\% } \\
\text { confidence interval) }\end{array}$ & \multicolumn{2}{c}{$\begin{array}{c}\text { Test of } \\
\text { isometry }\end{array}$} \\
\cline { 6 - 9 } & & & & & F & $p$ \\
\hline & S & 0.529 & $<0.001$ & $1.568(1.206,2.040)^{\mathrm{a}}$ & $-0.358(-0.705,-0.011)$ & 12.865 & 0.001 \\
& $\mathrm{SE}$ & 0.217 & 0.010 & $1.085(0.776,1.519)^{\mathrm{a}}$ & $-0.533(-0.899,-0.166)$ & 0.241 & 0.628 \\
& $\mathrm{E}$ & 0.121 & 0.060 & $1.805(1.265,2.576)^{\mathrm{ab}}$ & $-0.034(-0.607,0.540)$ & 12.458 & 0.001 \\
BGB- & $\mathrm{NE}$ & 0.617 & $<0.001$ & $1.693(1.335,2.147)^{\mathrm{ab}}$ & $-0.046(-0.426,0.334)$ & 22.195 & $<0.001$ \\
AGB & $\mathrm{N}$ & 0.389 & $<0.001$ & $0.902(0.670,1.215)^{\mathrm{ab}}$ & $-0.553(-0.803,-0.303)$ & 0.488 & 0.491 \\
& $\mathrm{NW}$ & 0.273 & 0.003 & $1.179(0.852,1.630)^{\mathrm{abc}}$ & $-0.426(-0.782,-0.070)$ & 1.048 & 0.315 \\
& $\mathrm{~W}$ & 0.377 & 0.001 & $1.651(1.199,2.723)^{\mathrm{bc}}$ & $0.245(-0.312,0.803)$ & 10.955 & 0.003 \\
& $\mathrm{SW}$ & 0.431 & $<0.001$ & $1.653(1.240,2.205)^{\mathrm{c}}$ & $-0.080(-0.544,0.385)$ & 13.536 & 0.001 \\
\hline & $\mathrm{S}$ & 0.174 & 0.022 & $0.715(0.507,1.010)$ & $-0.913(-1.132,-0.693)^{\mathrm{a}}$ & 3.951 & 0.057 \\
& $\mathrm{SE}$ & 0.155 & 0.031 & $1.030(0.727,1.450)$ & $-0.688(-1.044,-0.332)^{\mathrm{ab}}$ & 0.029 & 0.867 \\
& $\mathrm{E}$ & 0.263 & 0.004 & $1.154(0.833,1.600)$ & $-0.459(-0.801,-0.117)^{\mathrm{ab}}$ & 0.788 & 0.382 \\
FB- & $\mathrm{NE}$ & 0.353 & 0.001 & $0.819(0.603,1.113)$ & $-0.764(-1.010,-0.519)^{\mathrm{a}}$ & 1.753 & 0.196 \\
VB & $\mathrm{N}$ & 0.406 & $<0.001$ & $1.156(0.862,1.552)$ & $-0.499(-0.800,-0.197)^{\mathrm{abc}}$ & 1.002 & 0.325 \\
& $\mathrm{NW}$ & 0.459 & $<0.001$ & $1.130(0.853,1.497)$ & $-0.439(-0.733,-0.146)^{\mathrm{bc}}$ & 0.781 & 0.384 \\
& $\mathrm{~W}$ & 0.241 & 0.009 & $1.148(1.807,1.631)$ & $-0.535(-0.940,0.129)^{\mathrm{c}}$ & 0.627 & 0.436 \\
& $\mathrm{SW}$ & 0.312 & 0.001 & $0.939(0.685,1.288)$ & $-0.710(-1.004,-0.416)^{\mathrm{c}}$ & 0.160 & 0.693 \\
\hline
\end{tabular}




\begin{tabular}{c|c|c|c|c|c|c|c}
\hline & $\mathrm{S}$ & 0.312 & $<0.001$ & $1.748(1.275,2.398)^{\mathrm{A}}$ & $0.472(-0.076,1.019)$ & 14.079 & 0.001 \\
& $\mathrm{SE}$ & 0.340 & 0.001 & $1.518(1.114,2.069)^{\mathrm{AB}}$ & $0.328(-0.186,0.841)$ & 7.824 & 0.009 \\
& $\mathrm{E}$ & 0.024 & 0.413 & $1.238(0.852,1.799)^{\mathrm{ABC}}$ & $-0.059(-0.528,0.409)$ & 1.331 & 0.258 \\
$\mathrm{~PB}-$ & $\mathrm{NE}$ & 0.542 & $<0.001$ & $1.471(1.135,1.906)^{\mathrm{AB}}$ & $0.204(-0.196,0.605)$ & 9.568 & 0.004 \\
$\mathrm{NPB}$ & $\mathrm{N}$ & 0.723 & $<0.001$ & $0.975(0.796,1.194)^{\mathrm{C}}$ & $-0.312(-0.503,-0.122)$ & 0.064 & 0.802 \\
& $\mathrm{NW}$ & 0.740 & $<0.001$ & $1.278(1.050,1.554)^{\mathrm{ABC}}$ & $-0.041(-0.286,0.203)$ & 6.603 & 0.016 \\
& $\mathrm{~W}$ & 0.705 & $<0.001$ & $1.034(0.828,1.291)^{\mathrm{C}}$ & $-0.202(-0.455,0.050)$ & 0.094 & 0.761 \\
& $\mathrm{SW}$ & 0.719 & $<0.001$ & $1.078(0.879,1.321)^{\mathrm{BC}}$ & $-0.170(-0.407,0.067)$ & 1.556 & 0.462 \\
\hline
\end{tabular}

BGB-AGB represents above- and below-ground biomass, FB-VB represents reproduction- and vegetation- growth biomass, PB-NPB represents photosynthesis- and non photosynthesis- growth biomass. The data represent the mean $\pm \mathrm{SE}(\mathrm{n}=30)$. Data with the same letters within the same column indicate a not significant difference at $p<0.05$ level.

\section{Growth mass and biomass increment from early vegetation stage to full bloom stage}

There were significant differences in plant height increase $(\mathrm{F}=3.574, p=0.001)$, stem biomass increment $(\mathrm{F}=5.421, p<0.001)$, and belowground biomass increment $(\mathrm{F}$ $=2.682, p=0.011) . \Delta \mathrm{PN}$ on slope $\mathrm{S}$ was significantly greater than those on slopes $\mathrm{SW}$, $\mathrm{NE}, \mathrm{W}$ and SE. $\triangle \mathrm{SB}$ on slope $\mathrm{S}$ was significantly greater than those on other slope aspects, except slope E. $\triangle \mathrm{LB}$ was not significantly different among the eight slope aspects. $\triangle \mathrm{TB}$ on slope $\mathrm{S}$ was remarkably greater than those on slopes $\mathrm{SW}$ and $\mathrm{W}$. Along the circumference of the mountain, from slope $S$ to slope $S W$, the increment in stem biomass, leaf biomass, total biomass, and the increase in leaf numbers showed a "W"like trend, while the increase in plant height and the increment in belowground biomass showed a wave shape. The increasing of plant height and the increment of belowground biomass showed different trends. From slope $\mathrm{S}$ to slope $\mathrm{SW}, \Delta \mathrm{PH}$ had two spikes and three troughs of the wave. $\triangle \mathrm{BGB}$ on slope $\mathrm{N}$ was significantly greater than those on other slopes, except slope W and NW (Fig. 6, Table 5).
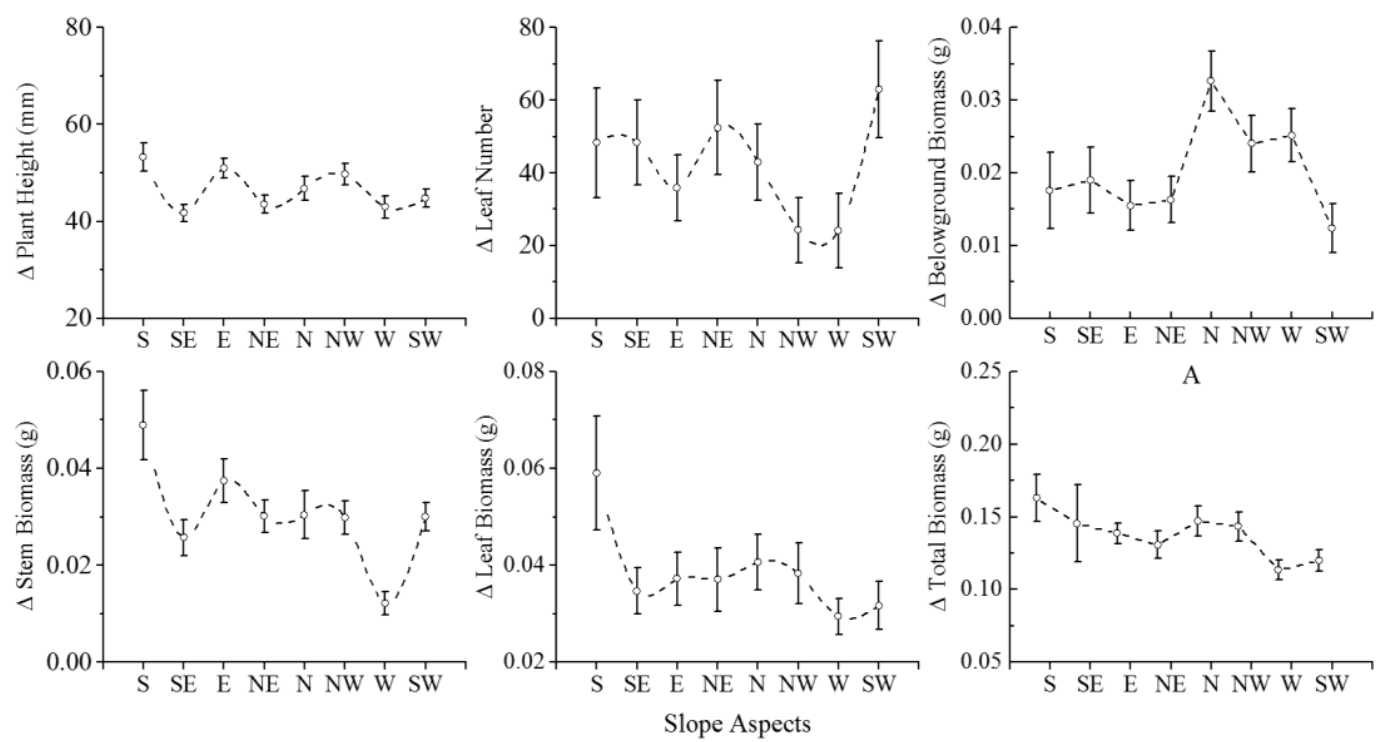

Figure 6. The growth mass and biomass increment from vegetation stage to full bloom stage on different slope aspects 
Table 5. Increase in plant height and biomass from the early vegetation stage to the full bloom stage among slope aspects

\begin{tabular}{c|c|c|c|c|c|c}
\hline $\begin{array}{c}\text { Slope } \\
\text { aspects }\end{array}$ & $\Delta \mathbf{P H}(\mathbf{m m})$ & $\Delta \mathbf{L N}$ & $\Delta \mathbf{S B}(\mathrm{g})$ & $\Delta \mathbf{L B}(\mathbf{g})$ & $\Delta \mathrm{BGB}(\mathrm{g})$ & $\Delta \mathrm{TB}(\mathrm{g})$ \\
\hline $\mathrm{S}$ & $53.27 \pm 2.86^{\mathrm{a}}$ & $48.27 \pm 15.11^{\mathrm{ab}}$ & $0.05 \pm 0.01^{\mathrm{a}}$ & $0.06 \pm 0.01$ & $0.02 \pm 0.01^{\mathrm{b}}$ & $0.17 \pm 0.02^{\mathrm{a}}$ \\
$\mathrm{SE}$ & $41.73 \pm 1.77^{\mathrm{d}}$ & $48.40 \pm 11.63^{\mathrm{ab}}$ & $0.03 \pm 0.00^{\mathrm{b}}$ & $0.07 \pm 0.04$ & $0.02 \pm 0.00^{\mathrm{b}}$ & $0.14 \pm 0.04^{\mathrm{ab}}$ \\
$\mathrm{E}$ & $51.00 \pm 2.07^{\mathrm{ab}}$ & $35.93 \pm 9.02^{\mathrm{ab}}$ & $0.04 \pm 0.00^{\mathrm{ab}}$ & $0.04 \pm 0.01$ & $0.02 \pm 0.00^{\mathrm{b}}$ & $0.13 \pm 0.01^{\mathrm{ab}}$ \\
$\mathrm{NE}$ & $43.53 \pm 1.93^{\mathrm{cd}}$ & $52.43 \pm 12.90^{\mathrm{ab}}$ & $0.03 \pm 0.00^{\mathrm{b}}$ & $0.04 \pm 0.01$ & $0.02 \pm 0.00^{\mathrm{b}}$ & $0.12 \pm 0.01^{\mathrm{ab}}$ \\
$\mathrm{N}$ & $46.80 \pm 2.46^{\mathrm{abcd}}$ & $42.97 \pm 10.56^{\mathrm{ab}}$ & $0.03 \pm 0.00^{\mathrm{b}}$ & $0.04 \pm 0.01$ & $0.03 \pm 0.00^{\mathrm{a}}$ & $0.15 \pm 0.02^{\mathrm{ab}}$ \\
$\mathrm{NW}$ & $49.77 \pm 2.20^{\mathrm{abc}}$ & $24.23 \pm 8.97^{\mathrm{b}}$ & $0.03 \pm 0.00^{\mathrm{b}}$ & $0.04 \pm 0.01$ & $0.02 \pm 0.00^{\mathrm{ab}}$ & $0.14 \pm 0.02^{\mathrm{ab}}$ \\
$\mathrm{W}$ & $42.96 \pm 2.32^{\mathrm{cd}}$ & $24.11 \pm 10.31^{\mathrm{b}}$ & $0.01 \pm 0.00^{\mathrm{c}}$ & $0.03 \pm 0.00$ & $0.03 \pm 0.00^{\mathrm{ab}}$ & $0.10 \pm 0.01^{\mathrm{b}}$ \\
$\mathrm{SW}$ & $44.77 \pm 1.86^{\mathrm{bcd}}$ & $62.93 \pm 13.36^{\mathrm{a}}$ & $0.03 \pm 0.00^{\mathrm{b}}$ & $0.03 \pm 0.01$ & $0.01 \pm 0.00^{\mathrm{b}}$ & $0.10 \pm 0.01^{\mathrm{b}}$ \\
$\mathrm{F}$ & 3.547 & 1.325 & 5.421 & 0.846 & 2.682 & 1.706 \\
$p$ & 0.001 & 0.239 & $<0.001$ & 0.55 & 0.011 & 0.108 \\
\hline
\end{tabular}

$\Delta \mathrm{PH}$ represent the increase in plant height, $\Delta \mathrm{LN}$ represent the increase in leaf number, $\Delta \mathrm{SB}$ represent the increase in stem biomass, $\Delta \mathrm{LB}$ represent the increase in leaf biomass, $\triangle \mathrm{BGB}$ represent the increase in belowground biomass and $\Delta \mathrm{TN}$ represent the increase in total biomass. The data represent the mean \pm $\mathrm{SE}(\mathrm{n}=30)$. Data with the same letters within the same column indicate a not significant difference at the $p<0.05$ level.

\section{Effect of environmental factors on plant characters and growth}

There were two principal components that were composed of environmental factors and had remarkable eigenvalues. The cumulative variance proportion explained by the first two principal components was $81.92 \%$. The first principal component (z1, 56.03\%) mainly consisted of soil organic carbon, soil total nitrogen and soil total phosphorus. The second principal component $(\mathrm{z} 2,25.89 \%)$ was composed of light intensity, mean annual temperature and soil water content (Fig. 7). As interpreted in Table 6, F/V was significantly correlated with the second component $\left(\mathrm{R}^{2}=0.717, p=0.008\right), \Delta \mathrm{SB}$ was significantly correlated with the first principal component $\left(\mathrm{R}^{2}=0.0 .537, p=0.039\right), \mathrm{R} / \mathrm{S}$ and $\triangle \mathrm{LB}$ were significantly marginally correlated with the first principal component $\left(\mathrm{R}^{2}\right.$ $\left.=0.464, p=0.063 ; \mathrm{R}^{2}=0.464, p=0.063\right)$, and $\Delta \mathrm{BGB}$ and $\Delta \mathrm{TB}$ were not significantly correlated with the principal component $\left(\mathrm{R}^{2}=0.350, p=0.122 ; \mathrm{R}^{2}=0.420, p=0.082\right)$ (Fig. 7, Table 6).

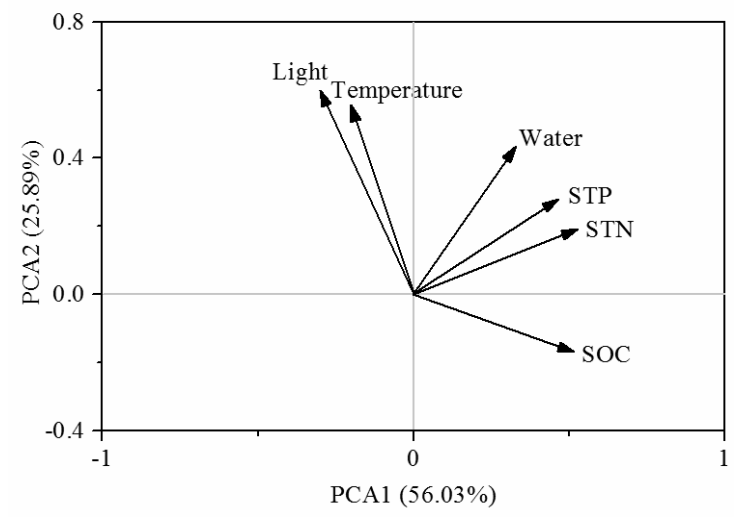


Figure 7. PCA ordination diagram of environmental factors. Light represents light intensity,

Water represents soil water content, Temperature represents mean annual temperature of soil, STP represents soil total phosphorus, STN represents soil total nitrogen and SOC represents soil organic carbon

Table 6. Stepwise regression analysis of plant traits and biomass with principal components

\begin{tabular}{c|c|c|c|c|c}
\hline \multirow{2}{*}{$\begin{array}{c}\text { Dependent } \\
\text { variable }\end{array}$} & \multicolumn{2}{|c|}{$\begin{array}{c}\text { Independent variable } \\
\text { (Partial R-Square) }\end{array}$} & \multirow{2}{*}{ Intercept } & \multirow{2}{*}{$\mathbf{R}^{2}$} & $\boldsymbol{p}$ \\
\cline { 2 - 3 } & $\mathbf{Z 1}$ & $\mathbf{Z 2}$ & & & \\
\hline $\mathrm{PH}$ & - & - & 68.175 & - & - \\
$\mathrm{R} / \mathrm{S}$ & $0.122(0.464)$ & - & 0.961 & 0.464 & 0.063 \\
$\mathrm{~F} / \mathrm{V}$ & - & $-0.016(0.717)$ & 0.259 & 0.717 & 0.008 \\
$\Delta \mathrm{PH}$ & - & - & - & - & - \\
$\Delta \mathrm{SB}$ & $-0.004(0.537)$ & - & 0.031 & 0.537 & 0.039 \\
$\Delta \mathrm{LB}$ & $-0.005(0.450)$ & - & 0.043 & 0.450 & 0.069 \\
$\Delta \mathrm{BGB}$ & $0.002(0.350)$ & - & 0.020 & 0.350 & 0.122 \\
$\Delta \mathrm{TB}$ & $-0.008(0.420)$ & - & 0.132 & 0.420 & 0.082 \\
\hline
\end{tabular}

\section{Discussion}

Effect of slope aspects on plant height, branch number, specific leaf area, leaf number and flower structure traits at full bloom stage

Plant height, branch number, specific leaf area and leaf number were significantly affected by slope aspect. Alpine plants change their survival strategy to adapt to different environmental conditions. Plant height is a sign of the ability of plants to compete for space resources (Westoby, 1998, 2002). This ability can be understood as a race upwards for the light. The plant height was highest on slope S, and was significantly higher than plant that on shady and partially sunny slopes. Plants in harsh locations tend to decrease their height to adapt to strong wind environments (Kudo, 1992; Chen, 2011). Studies haven shown that decreasing plant height plays an important role in avoiding the risk of shady slope with less light radiation, low temperature and strong wind (Körner and Neumayer, 1989; Méndez and Traveset, 2003). Plants prefer to grow shorter to reduce energy loss (Körner, 2003; Fabbro, 2004). At the same time, a reduction in plant size along elevational gradients can explain this question from other aspects (Körner et al., 1989; Fabbro, 2004; Fan, 2009). Leaf number is critical for the development of plants to enhance photosynthetic efficiency (Li et al., 2015), but light intensity among the eight slope aspects was not significantly different, so there was no significant difference between sunny slope and shady slope in leaf number. Increasing branch number is a way for asexual reproduction, which would be important for alpine plants as an adaption to harsh environments (He et al., 2017). In our results, the branch number of the plants on slope $\mathrm{N}$ was remarkably greater than that on the partial shady slope, slope E and slope $\mathrm{W}$, which indicated that the shady slope was conducive to asexual reproduction. Bonser et al. (1994) reported that plants tend to increase branch number when solar radiation is strong, which is opposite to our results. There were more diverse communities of pollinators visiting low elevation populations than high elevation populations (Bingham and Orthner, 1998; Arroyo, 1982). The climatic conditions of the shady slope were severe compared to those of the sunny slope, which was similar to the comparison of high elevation to low elevation, as plants prefer to 
increase investment to the propagules of asexual reproduction for population stability. The reasons may be that specific leaf area (SLA) can have large variability in response to irradiance, temperature, and water availability (Pigliucci and Kolodynska, 2002; Poorter et al., 2009). Specific leaf area is related to photosynthetic capacity and refers to the ability to acquire resources (Reich et al., 1997, 1999; Wright et al., 2004), which is associated with plant survival strategy. Many studies have shown that the lower SLA tends to be related more to lower leaf water content than to higher leaf thickness for herbaceous leaves with sclerophyllous leaves growing in a low temperature environment (Dijkstra and Lambers, 2010; Garnier and Laurent, 1994; Arendonk and Poorter, 1994; Shipley, 1995; Ryser and Aeschlimann, 1999). In our experiment, soil total nitrogen was higher on slopes W and SW, so SLA of Gentiana hexaphylla ion slopes SW and W was larger than on other slope aspects. For this reason, this finding was consistent with the research that species with tender leaves have few cell walls per unit leaf area and a high proportion of their volume is occupied by nitrogen-rich protoplasts (Reich, 1993; Garnier and Laurent, 1994; Vendramini et al., 2002). The carbon assimilation ability of these species was strong.

Slope aspect significantly affects flower structure traits including corolla length, calyx length, pistil length and stamen length. As the reproductive organs of plants, flowers play an indispensable role in protecting the genes of continuity and supporting genetic diversity. Flower size was closely related to pollinator visitation (Fenster et al., 2004). The vast majority of plants receive a variety of different species as visitors (Fenster et al., 2004). Meanwhile, flowers are the key factor affecting the evolution of plants and the guarantee of sexual reproduction (Goodwillie et al., 2010). The greater the flower number, the better is the possibility of producing offspring. Bynum (2001) says that Gentiana hexaphylla shows a low probability of autogamy because of the temporal and spatial separation of male and female reproduction traits, indicating the importance of pollinators for outcrossing. The experiments of Nilsson (1988) and Johnson and Steiner (1997) suggested that the reduction in corolla tube reduces reproductive success. In our study, corolla and calyx length were greater on slope SW than on slopes S and SE, and the lowest on slopes N and NW. Slope SW had a high rate of reproductive success. Above all, Gentiana hexaphylla grows in shady slopes, increasing asexual reproduction and investment of male organs to compensate for the influence of harsh environments of sexual reproduction in alpine ecosystems.

\section{Effect of abiotic environmental factors on resource allocation patterns and allometric relationships in alpine plants on different slope aspects}

Leaf biomass partitioning and flower biomass partitioning on different slope aspects did not significantly differ, but there were significant effects on other organ biomass partitioning. Plants adjusted their functional traits and biomass allocation to adapt to a harsh habitat when subjected to environmental stress or resource constraints to improve competitiveness and survival fitness (Sultan, 1992). Niklas and Enquist (2001) reported an isometric relationship between belowground and aboveground biomass across nonwoody plants. The resulting, belowground /aboveground biomass, had an allometric relationship, which means that the accumulating rate of underground biomass was greater than the rate of aboveground biomass. This result was opposite to that of Niklas and Enquist Most allocation issues were triggered by allometric growth, which can also be used to quantify the relationships between growth and partitioning (Weiner, 2004). To adapt to the harsh ecological environment in alpine ecosystems, more biomass is 
allocated to the underground to produce fleshy roots. In this study, photosynthetic /nonphotosynthetic biomass growth showed that an allometric relationship expressed photosynthetic biomass accumulation faster than that of non-photosynthetic biomass. At the same time, photosynthetic biomass on the sunny slopes was significantly greater than those on the shady slopes and slope W, and there was no significant difference in leaf biomass allocation among the different slopes. Plants were prone to make full use of light conditions and increased area of photosynthesis for increasing plant adaptability to the environment (Körner, 2003). Leaf biomass partitioning was a rather conserved trait in cold high elevation climate regions. However, alpine plants tend to invest more biomass in photosynthetic tissue for growth and persistence than do herbaceous species in cold habitats (Körner, 2003; Patty et al., 2010). There was a significant correlation between R/S and soil nutrients. Soil organic carbon, total nitrogen and phosphorus on slope $\mathrm{W}$ were highest and these on slope $\mathrm{S}$ were lowest. The root biomass and proportion of biomass allocation among different slopes had the same trend as soil nutrients. In the experimental area, site $\mathrm{E}$ was the windward slope and site $\mathrm{W}$ was the leeward slope. Long-term operating soil weathering and erosion processes accelerated lower soil nutrients in shady slopes and slope E (Rech et al., 2001). Northern slopes exhibited a higher productivity and water content was an important limiting factor for plant growth (Gong et al., 2008), which was opposite to our results. The annual direct radiation on slope $\mathrm{S}$ was higher than on slopes SW, W and $\mathrm{N}$ (McCune and Keon, 2002). Higher radiation resulted in higher soil temperature (McCune and Keon, 2002), which led to faster decomposition of soil organic matter, meaning that slope $S$ accumulated less soil organic carbon than did slopes SW, N and W. Thus, plants growing on slope west-facing slope and shady slopes inclined to increase belowground biomass, while plants growing on sunny slopes were more conductive to increase aboveground biomass.

\section{The relationship between plant growth from the early vegetation stage to the full bloom stage and environmental factors on different slope aspects}

Slope aspect was an important topographic variable that substantially modified light radiation, soil temperature and soil water content, influencing the ecological processes and creating microclimates that differ significantly from the regional climatic conditions (Buffo et al., 1972; Flint and Childs, 1987; Nikolov and Zeller, 1992; McCune and Keon, 2002; Bennie et al., 2008; Zhang et al., 2015). The micro-environmental conditions on different aspects may have marked effects on the growth and development of the plants ( $\mathrm{Li}$ et al., 2013). There was a significant relationship between growth and environmental factors composed of light intensity, mean annual temperature and soil water content. The sunny slopes had a larger light intensity and higher mean annual temperature, which was more beneficial for herbs to grow higher and increase biomass (Poorter et al., 2012). The reason why plants grown on slope $\mathrm{N}$ were larger than those grown on partially shady slopes may be associated with soil water content. Gong et al. (2008) showed that water was an important factor for plant growth. Gentiana hexaphylla adopted a different growth strategy. Soil organic carbon, soil total nitrogen and soil total phosphorus were the primary factors influencing the increment from the early vegetation stage to the full bloom stage. Soil nutrients are restrictive factors for plant growth, affecting the material circulation of plants (Sterner and Elser, 2002). The herbs growing on the slope contained more nutrients and were prone to 
increase root biomass, and those growing in lower nutrient places tend to increase aboveground biomass.

\section{Conclusion}

Slope aspect had marked effects on the growth and reproduction of Gentiana hexaphylla. The plants grown on a shady slope inclined to increase belowground biomass, while plants grown on a sunny slope were more conductive to increase aboveground biomass. This phenomenon was more related to soil organic carbon, soil total nitrogen and phosphorus than to light intensity, soil temperature, and soil water content. Gentiana hexaphylla inclined to increase sexual reproduction investment on sunny slopes and increase male organ input and asexual reproduction on shady slope. Along the circumference of the mountain, from slope $\mathrm{S}$ to slope SW, the growth of plant height from the early vegetation stage to the full bloom stage showed a wave shape, and the increment in organ biomass showed a "W" shape.

Acknowledgements. We thank Chen Zukang and Zhang Baozhen for assistance in the field. This study was funded by National Key R\&D Program of China (2017YFC0505005-1), the National Natural Science Foundation of China (No. 31400389, 41661144045).

\section{REFERENCES}

[1] Ai, Z. M., He, L. R., Xin, Q., Yang, T., Liu, G. B., Xue, S. (2017): Slope aspect affects the non-structural carbohydrates and $\mathrm{C}: \mathrm{N}$ : P stoichiometry of Artemisia sacrorum on the Loess Plateau in China. - Catena 152: 9-17.

[2] Allen, S. E. (1989): Chemical Analysis of Ecological Material. - Blackwell Scientific Publications, Oxford.

[3] Arendonk, J. J. C. M. van, Poorter, H. (1994): The chemical composition and anatomical structure of leaves of grass species differing in relative growth rate. - Plant, Cell \& Environment 17(8): 963-970.

[4] Arroyo, M. T. K., Primack, R. B., Armesto, J. J. (1982): Community studies in pollination ecology in the high temperate Andes of central Chile. I. Pollination mechanisms and altitudinal variation. - American Journal of Botany 69(1): 82-97.

[5] Auslander, M., Nevo, E., Inbar, M. (2003): The effects of slope orientation on plant growth, developmental instability and susceptibility to herbivores. - Journal of Arid Environments 55(3): 405-416.

[6] Bao, S. D. (2000): Soil and Agricultural Chemistry Analysis (in Chinese). - China Agriculture Press, Beijing.

[7] Barthlott, W., Lauer, W., Placke, A. (1996): Species diversity in vascular plants: towards a world map of phytodiversity. - Erdkunde 50(4): 317-327.

[8] Bennie, J., Huntley, B., Wiltshire, A., Hill, M. O., Baxter, R. (2008): Slope, aspect and climate: Spatially explicit and implicit models of topographic microclimate in chalk grassland. - Ecological Modelling 216(1): 47-59.

[9] Bingham, R. A., Orthner, A. R. (1998): Efficient pollination of alpine plants. - Nature 391(6664): 238-239.

[10] Bonser, S. P., Aarssen, L. W. (1994): Plastic allometry in young sugar maple (AcerSaccharum): adaptive responses to light availability. - American Journal of Botany 81(4): 400-406. 
[11] Bonser, S. P., Aarssen, L. W. (2009): Interpreting reproductive allometry: Individual strategies of allocation explain size-dependent reproduction in plant populations. Perspectives in Plant Ecology, Evolution and Systematics 11(1): 31-40.

[12] Buffo, J., Fritschen, L. J., Murphy, J. L. (1972): Direct Solar Radiation on Various Slopes from 0 to 60 Degrees North Latitude. - Pacific Northwest Forest and Range Experiment Station, Portland, OR.

[13] Bynum, M. R., Smith, W. K. (2001): Floral movements in response to thunderstorms improve reproductive effort in the alpine species Gentiana algida (Gentianaceae). American Journal of Botany 88(6): 1088-1095.

[14] Caldwell, M. M. (1968): Solar ultraviolet radiation as an ecological factor for alpine plants. - Ecological Monographs 38(3): 243-268.

[15] Caldwell, M. M. (1979): Plant life and ultraviolet radiation: Some perspective in the history of the Earth's UV climate. - Bioscience 29(9): 520-525.

[16] Cantón, Y., Del Barrio, G., Sole-Benet, A., Lazaro, R. (2004): Topographic controls on the spatial distribution of ground cover in the Tabemas badlands of SE Spain. - Catena 55(3): 341-365.

[17] Carletti, P., Vendramin, E., Pizzeghello, D., Concheri, G., Zanella, A., Nardi, S., Squartini, A. (2009): Soil humic compounds and microbial communities in six spruce forests as function of parent material, slope aspect and stand age. - Plant and Soil 315(12): 47-65.

[18] Chen, W. N., Wu, Y., Wu, N., Luo, P. (2008): Effect of snow-cover duration on plant species diversity of alpine meadows on the eastern Qinghai-Tibetan Plateau. - Journal of Mountain Science 5(4): 327-339.

[19] Chen, W. N., Wu, Y., Wu, N., Wang, Q. (2011): Effect of snowmelt time on growth and reproduction of Pedicularis davidiivar pentodon in the eastern Tibetan Plateau. - Plant Biosystems - An International Journal Dealing with all Aspects of Plant Biology 145(4): 802-808.

[20] De Bello, F., Leps, J., Sebastia, M. T. (2006): Variations in species and functional plant diversity along climatic and grazing gradients. - Ecography 29(6): 801-810.

[21] Dijkstra, P., Lambers, H. (2010): Analysis of specific leaf area and photosynthesis of two inbred lines of Plantago mayor differing in relative growth rate. - New Phytologist 113(3): 283-290.

[22] Fabbro, T., Körner, C. (2004): Altitudinal differences in flower traits and reproductive allocation. - Flora 199(1): 70-81.

[23] Fan, D. M., Yang, Y. P. (2009): Altitudinal variations in flower and bulbil production of an alpine perennial, Polygonum viviparum L. (Polygonaceae). - Plant Biology 11(3): 493497.

[24] Fekedulegn, D., Hicks, R. R., Colbert, J. J. (2003): Influence of topographic aspect, precipitation and drought on radial growth of four major tree species in an Appalachian watershed. - Forest Ecology and Management 177(1): 409-425.

[25] Fenster, C. B., Armbruster, W. S., Wilson, P., Dudash, M. R., Thomson, J. D. (2004): Pollination syndromes and floral specialization. - Annual Review of Ecology Evolution and Systematics 35(35): 375-403.

[26] Flint, A. L., Childs, S. W. (1987): Calculation of solar radiation in mountainous terrain. Agricultural and Forest Meteorology 40(3): 233-249.

[27] Garnier, E., Laurent, G. (1994): Leaf anatomy, specific mass and water content in congeneric annual and perennial grass species. - New Phytologist 128(4): 725-736.

[28] Gong, X., Brueck, H., Giese, K. M., Zhang, L., Sattelmacher, B., Lin, S. (2008): Slope aspect has effects on productivity and species composition of hilly grassland in the Xilin River Basin, Inner Mongolia, China. - Journal of Arid Environments 72(4): 483-493.

[29] Goodwillie, C., Sargent, R. D., Eckert, C. G., Elle, E., Geber, M. A., Johnston, M. O., Kalisz, S., Moeller, D. A., Ree, R. H., Vallejo-Marin, M., Winn, A. A. (2010): Correlated evolution of mating system and floral display traits in flowering plants and its 
implications for the distribution of mating system variation. - New Phytologist 185(1): 311-321.

[30] Grassein, F., Till-Bottraud, I., Lavorel, S. (2010): Plant resource-use strategies: the importance of phenotypic plasticity in response to a productivity gradient for two subalpine species. - Annals of Botany 106(4): 637-645.

[31] He, J. D., Xue, J. Y., Gao, J., Wang, J. N., Wu, Y. (2017): Adaptations of the floral characteristics and biomss allocation patterns of Gentiana hexaphylla to the altitudinal gradient of the eastern Qinghai-Tibet Plateau. - Journal of Mountain Science 14(8): 1563-1576.

[32] He, Y. P., Duan, Y. W., Liu, J. Q., Smith, W. K. (2005): Floral closure in response to temperature and pollination in Gentiana straminea Maxim. (Gentianaceae), an alpine perennial in the Qinghai-Tibetan Plateau. - Plant Systematics and Evolution 256(1-4): 17-33.

[33] IPCC (2001): Third Assessment Report of Working Group II. - Climate Change 2001: Impacts, Adaptation and Vulnerability. - Cambridge University Press, Cambridge.

[34] Johnson, S. D., Steiner, K. E. (1997): Long-tongued fly pollination and evolution of floral spur length in the Disa draconis complex (Orchidaceae). - Evolution 51(1): 45-53.

[35] Kareiva, P. M., Kingsolver, J. G., Huey, R. B. (1993): Biotic Interactions and Global Change. Patterns and Determinants of Climate and Landscape Change: Scenarios of Global Warming. - Sinsuer Associates Inc., Sunderland, MA.

[36] Körner, C. (2003): Alpine Plant Life: Functional Plant Ecology of High Mountain Ecosystems. - Springer-Verlag, Heidelberg.

[37] Körner, C., Neumayer, M., Menendez-Riedl, S. P., Smeets-Scheel, A. (1989): Functional morphology of mountain plants. - Flora 182: 353-383.

[38] Kudo, G. (1992): Performance and phenology of alpine herbs along a snow-melting gradient. - Ecological Research 7(3): 297-304.

[39] Li, D., Wang, X. F., Zhang, X. B., Chen, Q. Y., Xu, G. H., Xu, D. Y., Wang, C. L., Liang, Y. M., Wu, L. S., Huang, C., Tian, J. G., Wu, Y. Y., Tian, F. (2016): The genetic architecture of leaf number and its genetic relationship to flowering time in maize. - New Phytologist 210(1): 256-268.

[40] Li, Q. H., Xu, J., Li, H. Q., Wang, S. X., Yan, X., Xin, Z. M., Jiang, Z. P., Wang, L. L., Jia, Z. Q. (2013): Effects of aspect on clonal reproduction and biomass allocation of layering modules of Nitraria tangutorum in nebkha dunes. - PLoS One 8(10): e79927.

[41] McCune, B., Keon, D. (2002): Equations for potential annual direct incident radiation and heat load. - Journal of Vegetation Science 13(4): 603-606.

[42] Mcintosh, M. (2002): Plant size, breeding system, and limits to reproductive success in two sister species of Ferocactus (Cactaceae). - Plant Ecology 162(2): 273-288.

[43] Nagy, L., Grabherr, G. (2009): The Biology of Alpine Habitats. - Oxford University Press, UK.

[44] Niklas, K. J. (2005): Modelling below- and above-ground biomass for non-woody and woody plants. - Annals of Botany 95(2): 315-321.

[45] Niklas, K. J., Enquist, B. J. (2001): Invariant scaling relationships for interspecific plant biomass production rates and body size. - Proceedings of the National Academy of Sciences of the United States of America 98(5): 2922-2927.

[46] Nikolov, N. T., Zeller, K. F. (1992): A solar radiation algorithm for ecosystem dynamic models. - Ecological Modeling 61(3-4): 149-168.

[47] Nilsson, L. A. (1988): The evolution of flowers with deep corolla tubes. - Nature 334(6178): 147-149.

[48] Ohba, H. (1988): The Alpine Flora of the Nepal Himalayas: An Introductory Note. The Himalayan Plants. - University of Tokyo Press, Tokyo.

[49] Patty, L., Halloy, S. R. P., Hiltbrunner, E., Körner, C. (2010): Biomass allocation in herbaceous plants under grazing impact in the high semi-arid Andes. - Flora 205(10): 695-703. 
[50] Peng, D. L., Zhang, Z. Q., Xu, B., Li, Z. M., Sun, H. (2012): Patterns of flower morphology and sexual systems in the subnival belt of the Hengduan Mountains, SW China. - Alpine Botany 122(2): 65-73.

[51] Pigliucci, M., Kolodynska, A. (2002): Phenotypic plasticity and integration in response to flooded conditions in natural accessions of Arabidopsis thaliana. - Annals of Botany 90(2): 199-207.

[52] Poorter, H., Niinemets, U., Poorter, L, Wright, I. J., Villar, R. (2009): Causes and consequences of variation in leaf mass per area (LMA): a meta-analysis. - New Phytologist 182(3): 565-588.

[53] Poorter, H., Niklas, K. J., Reich, P. B., Oleksyn, J., Poot, P., Mommer, L. (2012): Biomass allocation to leaves, stems and roots: meta-analyses of interspecific variation and environmental control. - New Phytologist 193(1): 30-50.

[54] Rech, J. A., Reeces, R. W., Hendricks, D. M. (2001): The influence of slope aspect on soil weathering processes in the Springerville volcanic field, Arizona. - Catena 43(1): 4962.

[55] Reich, P. B. (1993): Reconciling apparent discrepancies among studies relating life span, structure and function of leaves in contrasting plant life forms and climates: 'The blind men and the elephant retold'. - Functional Ecology 7(6): 721-725.

[56] Reich, P. B., Walters, M. B., Ellsworth, D. S. (1997): From tropics to tundra: global convergence in plant functioning. - Proceedings of the National Academy of Sciences 94(25): 13730-13734.

[57] Reich, P. B., Ellsworth, D. S., Walters, M. B., Vose, J. M., Gresham, C., Volin, J. C., Bowman, W. D. (1999): Generality of leaf train relationships: a test across six biomes. Ecology 80(6): 1955-1969.

[58] Rosenberg Rosenberg, N. J., Blad, B. L., Verma, S. B. (1983): Microclimate-The Biological Environment. - Wiley, Chichester.

[59] Ryser, P., Aeschlimann, U. (1999): Proportional dry-mass content as an underlying trait for the variation in relative growth rate among 22 Eurasian populations of Dactylis glomeratas L. - Functional Ecology 13(4): 473-482.

[60] Shipley, B. (1995): Structured interspecific determinants of specific leaf area in 34 species of herbaceous angiosperms. - Functional Ecology 9(2): 312-319.

[61] Sternberg, M., Shoshany, M. (2001): Influence of slope aspect on Mediterranean woody formations: comparison of a semiarid and an arid site in Israel. - Ecological Research 16(2): 335-345.

[62] Sterner, R. W., Elser, J. J. (2002): Ecological stoichiometry: The biology of elements from molecules to the biosphere, pp. 167-196. - Princeton University Press, Princeton.

[63] Stöcklin, J. (1992): Umwelt, Morphologie und Wachstumsmuster klonaler Pflanzen: eine Übersicht. - Botanica Helvetica 102: 3-21.

[64] Sultan, S. E. (1992): Phenotypic plasticity and the neo-Darwinian legacy. - Evolutionary Trends in Plants 6(2): 61-71.

[65] Sun, H., Niu, Y, Chen, Y. S., Song, B., Liu, C. Q., Peng, D. L., Chen, J. G., Yang, Y. (2014): Survival and reproduction of plant species in the Qinghai-Tibet Plateau. - Journal of Systematics and Evolution 52(3): 378-396.

[66] Vendramini, F., Diaz, S., Gurvich, D. E., Wilson, P. J., Thompson, K., Hodgson, J. G. (2002): Leaf traits as indicators of resource-use strategy in floras with succulent species. - New Phytologist 154(1): 147-157.

[67] Vile, D., Garnier, E., Shipley, B., Laurent, G., Navas, M. L., Roumet, C., Lavorel, S., Diaz, S., Hodgson, J. G., Lloret, F., Midgley, G. F., Poorter, H., Rutherford, M. C., Wilson, P. J., Wright, I. J. (2005): Specific leaf area and dry matter content estimate thickness in laminar leaves. - Annals of Botany 96(6): 1129-1136.

[68] Warton, D. I., Wright, I. J., Falster, D. S., Westoby, M. (2006): Bivariate line-fitting methods for allometry. - Biological Reviews of the Cambridge Philosophical Society 81(2): 259-291. 
[69] Weiner, J. (2004): Allocation, plasticity and allometry in plants. - Perspectives in Plant Ecology, Evolution and Systematics 6(4): 207-215.

[70] Westoby, M. (1998): A leaf-height-seed (LHS) plant ecology strategy scheme. - Plant and Soil 199(2): 213-227.

[71] Westoby, M., Falster, D. S., Moles, A. T., Vesk, P. A., Wright, I. J. (2002): Plant ecological strategies: Some leading dimensions of variation between species. - Annual Review of Ecology and Systematics 33(1): 125-159.

[72] Wright, I. J., Reich, P. B., Westoby, M. (2001): Strategy shifts in leaf physiology, structure and nutrient content between species of high- and low-rainfall and high- and low-nutrient habitats. - Functional Ecology 15(4): 423-434.

[73] Wright, I. J., Reich, P. B., Westoby, M., Ackerly, D. D., Baruch, Z., Bongers, F., Cavender-Bares, J., Chapin, T., Cornelissen, J. H., Diemer, M., Flexas, J., Garnier, E., Groom, P. K., Gulias, J., Hikosaka, K., Lamont, B. B., Lee, T., Lee, W., Lusk, C., Midgley, J. J., Navas, M. L., Niinemets, U., Oleksyn, J., Osada, N., Poorter, H., Poot, P., Prior, L., Pyankov, V. I., Roumet, C., Thomas, S. C., Tjoelker, M. G., Veneklaas, E. J., Villar, R. (2004): The worldwide leaf economics spectrum. - Nature 428(6985): 821-827.

[74] Yang, Y., Sun, H. (2006): Advances in the functional ecology of alpine and arctic plants. - Acta Botanica Yunnanica 28(1): 43-53.

[75] Zhang, Y. L., Li, X., Bai, Y. L. (2015): An integrated approach to estimate shortwave solar radiation on clear-sky days in rugged terrain using modis atmospheric products. Solar Energy 113: 347-357. 